\title{
Adenoide lieber beobachten statt operieren
}

\section{Zumindest bei holländischen Klein- kindern mit rezidivierenden Infekti- onen der oberen Luftwege führt die Zeit, nicht aber die Entfernung der Rachenmandeln zu einem Rückgang der Infektionen.}

- Die Adenoidektomie gehört in den westlichen Industrieländern mit zu den häufigsten Operationen bei Kleinkindern. In einer offenen randomisierten und kontrollierten Studie wurden 111 Kinder im Alter zwischen ein und sechs Jahren mit rezidivierenden Infekten der oberen Luftwege und vergröBerten Rachenmandeln entweder sofort einer Adenoidektomie mit und ohne Tympanozentese zugeführt oder nur abwartend beobachtet. Im Verlauf einer medialen Beobachtungszeit von zwei Jahren kam es bei den operierten Kindern durchschnittlich zu 7,91 Episoden eines Infekts der oberen Luftwege pro Person im Jahr, in der Beobachtungsgruppe waren es 7,84 Episoden. Bei den sekundären Parametern wie derAnzahl der Tage mit Infekten, Mittelohrentzündungen und der allgemeinen Lebensqualität bestand zwischen den beiden Gruppen kein signifikanter Unterschied. Die Zahl der Fiebertage war nach der Adenoidektomie sogar signifikant höher als in der Beobachtungsgruppe.

Diese Tatsache bestätigt die Hypothese, wonach die Rachenmandeln eine gewisse Funktion in der Immunabwehr haben. Gegen eine vorschnelle Operation spricht auch die Beobachtung, dass die Zahl der Infekte der oberen Luftwege mit zunehmendem Lebensalter ohnehin abnahm.

\section{Kommentar}

Die Studie zeigt eindrucksvoll, dass es besser ist, Adenoide nur zu beobachten, da man von ihrer Entfernung keinen Nutzen erwarten kann, zumindest was die Häufigkeit von Infekten der oberen Luftwege angeht. Ob die Untersuchung auch auf Deutschland oder andere Länder zu übertragen ist, erscheint allerdings fraglich, da in den Niederlanden ganz ungewöhnlich hohe
Raten von Adenoidektomien üblich sind. Bei 0 - bis 4-jährigen Kindern wird die Operation in einer Prävalenz von 16,3/1000/Jahr, bei den 5- bis 9-jährigen Kindern von 5,5/1000/ Jahr durchgeführt. Entsprechende Zahlen aus den USA liegen bei nur 1,76/1000/Jahr. Um derartige Daten richtig interpretieren zu können, müssen die Versorgungsstruk- turen, Behandlungsgewohnheiten und die Vergütungssysteme in einzelnen Ländern mit berücksichtigt werden.

H. S. FüESSL =

- M. T. A. van den Artweg et al.

Effectiveness of adenoidectomy in children with recurrent upper respriratory tract infections: open randomised controlled trial. Brit. Med. J. 343 (2011) d5154

\section{Achtung:}

\section{Hier muss der Dummy durch eine Anzeige ersetzt werden !!}

